in space Research

Elsevier Editorial System(tm) for Advances

Manuscript Draft

Manuscript Number:

Title: Calculation of atmospheric ionization induced by electrons with non - vertical precipitation: updated model CRAC-EPII

Article Type: EM -Earth Magnetosphere/Upper Atmosphere

Keywords: Relativistic Electron Precipitation (REP), ionization of the Earth

atmosphere by electrons and energetic bremsstrahlung, monoenergetic electrons with

relativistic energies, angular distributions of relativistic electron precipitation

Corresponding Author: Dr. Anton Artamonov,

Corresponding Author's Institution: University of Oulu

First Author: Anton Artamonov

Order of Authors: Anton Artamonov; Irina Mironova; Gennady Kovaltsov; Alexander Mishev; Evgenii Plotnikov; Natalia Konstantinova

Abstract: In this paper we present a method to compute ionization rates induced by relativistic

electron precipitation with non-vertical incidence. Atmospheric

ionization for monoenergetic

$(>100 \mathrm{keV})$ relativistic electron precipitation including explicitly

ionization via

bremsstrahlung radiation is considered. Two peaks of energy deposition can be identified

for the ionization profiles caused by relativistic electrons. The first ionization peak is

related to direct ionization of primary relativistic electrons and the second corresponds

to bremsstrahlung radiation. The ionization rates are presented in Lookup Tables for

vertical, isotropic and angular distributions as well as with 15, 30 and 45 angles of

electron incidences. A computation scheme is provided to compute

ionization for an

arbitrary angular distribution of precipitation electrons.

Suggested Reviewers: Galina Bazilevskaya

Lebedev Physics Institute, Russian Academy of Sciences

gbaz@rambler.ru

Lachezar Mateev

Space Research and Technology Institute, Bulgarian Academy of Sciences Inmateevabas.bg

Vladimir Makhmutov

Lebedev Physics Institute, Russian Academy of Sciences 
makhmutv@lebedev.ru

Keri Nicoll

Department of Meteorology, University of Reading k.a.nicolldreading.ac.uk

Karen Aplin

University of Oxford, Department of Physics k.aplinl@physics.ox.ac.uk

Giles Harrison

Department of Meteorology, University of Reading r.g.harrisondreading.ac.uk

Esa Turunen

Sodankylä Geophysical Observatory etesgo.fi

Annika Seppala

Finnish Meteorological Institute annika.seppala@fmi.fi 


\title{
Calculation of atmospheric ionization induced by electrons with non - vertical precipitation: updated model CRAC-EPII
}

\author{
Anton Artamonov ${ }^{a *}$, Irina Mironova $^{b}$, Gennady Kovaltsov $^{c}$, Alexander Mishev $^{a}$, \\ Evgenii Plotnikov $^{d}$, Natalia Konstantinova ${ }^{e}$ \\ a Space Climate Research Unit, University of Oulu, Finland \\ ${ }^{b}$ St. Petersburg State University, Institute of Physics, St. Petersburg, Russia \\ ${ }^{c}$ Ioffe Physical-Technical Institute, St. Petersburg, Russia \\ ${ }^{d}$ National Research Tomsk Polytechnic University, Tomsk, Russia \\ ${ }^{e}$ State Scientific Center of the Russian Federation -Institute of Biomedical Problem of the RAS, Moscow, \\ Russia
}

\begin{abstract}
In this paper we present a method to compute ionization rates induced by relativistic electron precipitation with non-vertical incidence. Atmospheric ionization for monoenergetic $(>100 \mathrm{keV})$ relativistic electron precipitation including explicitly ionization via bremsstrahlung radiation is considered. Two peaks of energy deposition can be identified for the ionization profiles caused by relativistic electrons. The first ionization peak is related to direct ionization of primary relativistic electrons and the second corresponds to bremsstrahlung radiation. The ionization rates are presented in Look-up Tables for vertical, isotropic and angular distributions as well as with $15^{\circ}, 30^{\circ}$ and $45^{\circ}$ angles of electron incidences. A computation scheme is provided to compute ionization for an arbitrary angular distribution of precipitation electrons.
\end{abstract}

Keywords: Relativistic Electron Precipitation (REP), ionization of the Earth atmosphere by electrons and energetic bremsstrahlung, monoenergetic electrons with relativistic energies, angular distributions of relativistic electron precipitation

\section{Introduction}

2 Observation of atmospheric disturbances induced by energetic electron precipitation 3 has a long story (Rees, 1969; Newell et al., 1996; Frahm et al., 1997; Newell et al., 2010).

4 Continuous effects of electron precipitation are observed in the auroral zone (Newell

5 et al., 1996, 2010). However relativistic electron precipitation (REP) can occur also in

\footnotetext{
*Corresponding author: Anton Artamonov (anton.artamonov@oulu.fi and anton.art.an@gmail.com) Preprint submitted to Advances in Space Research 
sub-auroral zone and middle latitudes, from $\mathrm{L}=1.5$ to $\mathrm{L}=8$ field line region corresponding to an annulus with a $15^{0}$ latitudinal width (Frahm et al., 1997, Cresswell-Moorcock et al., 2013; Makhmutov et al., 2016; Remenets and Astafiev, 2015; Shprits et al., 2016). Here we focus on electrons in the energy range $100 \mathrm{keV}-500 \mathrm{MeV}$. Typically, the primary ionization is induced via direct electron impact and secondary is mostly due to bremsstrahlung. These sources of induced ionization are important because of possible implications for the chemistry of the middle atmosphere (Mironova et al., 2015; Matthes et al., 2016).

Energetic electrons interact in the lower ionosphere where they produce secondary electrons and create an impulsive ionization enhancement. As a result, the ionospheric conductivity is greatly enhanced. REP approach also lower altitudes and leads to enhance ionization forming a disturbance of the Earth's ionosphere through VLF signal propagation. Generally, such electrons are detected by balloon measurements (Makhmutov et al., 2016) and/or with multi-frequency VLF radio signals detectors (Remenets and Astafiev, 2015, 2016).

Satellite-based measurements do not allow to measure precipitating electrons in a wide energy range e.g. from hundred $\mathrm{keV}$ to $\mathrm{MeV}$, but observations of the height-resolved electron density profiles at night give proxy for precipitating electrons in the middle energy range (Miyoshi et al., 2015, Kero et al. 2014). Thus as discussed in Miyoshi et al. (2015) the stopping heights of 100,200 , and $400 \mathrm{keV}$ electrons being 80,70, and $60 \mathrm{~km}$, respectively. Turunen et al. (2009) showed that the stopping height for monoenergetic beams from $4 \mathrm{keV}$ to $10 \mathrm{MeV}$ electrons is from 120 to $40 \mathrm{~km}$. However all these works did not discuss energies of relativistic electron precipitation and additional ionization induced by bremsstrahlung.

In this paper we present atmospheric profiles of ionization rates induced by relativistic electron precipitation taking into account ionization induced by bremsstrahlung. We also present a computation scheme of ionization rates for vertical, isotropic and angular distributions of REP, we consider electron energies from $100 \mathrm{keV}$ to $500 \mathrm{MeV}$ on the reason of the modern measurements that showed energies of REP to about $4 \mathrm{MeV}$ (Shprits et al. 2016) and even greater than $10 \mathrm{MeV}$ (Makhmutov et al., 2016; Remenets and Astafiev, 2015, 2016). Here we employ precomputed ionization yield function of REP with vertical incidence, which were simulated by Monte Carlo tools (Artamonov et al. 2016; Artamonov et al. 2016b). The ionization rates as functions for precipitating electrons with vertical and isotropic angular distribution as well as with $15^{\circ}, 30^{\circ}$ and $45^{\circ}$ angles of electron incidence are given in Look-up Tables, see Supplementary materials and Appendix of this paper. 


\section{Scheme of computation of ionization rates induced by REP with different angle distribution of electrons' incidence}

The ionization yield function $Y(x, K)$ (ion pairs $\mathrm{cm}^{2} \mathrm{~g}^{-1}$ ) is a number of ion pairs induced by precipitating electron with given energy. The ionization yield function $Y(x, K)$ is computed per one gram of atmosphere at depth $x\left(\mathrm{~g} \mathrm{~cm}^{-2}\right)$ and per one precipitating electron with initial kinetic energy $K$ at the upper boundary of atmosphere (Artamonov et al., 2016). For vertical incident of electron precipitation, ionization yield function $Y_{v}(x, K)$ was computed (Artamonov et al., 2016). This vertical ionization yield function allows to compute ionization of atmosphere induced by energetic electron precipitation with one any angular distribution at the upper boundary of atmosphere. For precipitating electron with zenith angle $\alpha$ at the upper boundary of atmosphere, ionization yield function $Y_{\alpha}(x, K)$ can be computed as :

$$
Y_{\alpha}(x, K)=Y_{v}\left(x,{ }^{\prime} K\right) / \cos \alpha,
$$

In section 3 we present results for angular distribution function:

$$
f(\alpha)=\frac{n+1}{2 \pi} \cos ^{n} \alpha .
$$

64 In the isotropic case $(n=1): f(\alpha)=(1 / \pi) \cos \alpha$. Isotropic case means isotropic distri65 bution of energetic particles outside of the Earth atmosphere, (see Banks et al. (1974);

66 Usoskin and Kovaltsov (2006); Usoskin et al. (2010)). Consideration of isotropic case 
is used for calculation of ionisation of atmosphere induced by different sources of particles: galactic cosmic rays (Bazilevskaya et al., 2008), solar energetic particles (Usoskin et al., 2011), energetic electrons (Goldberg et al., 1984; Turunen et al., 2009).

Ionization rates (ion pairs per $\mathrm{cm}^{-3} \mathrm{~s}^{-1}$ ) at atmospheric altitude $h(\mathrm{~km})$ can be computed as:

$$
I(h, K)=Y(x, K) \rho(h) F(K),
$$

where $\rho(h)$ is the density of the atmosphere at a given altitude $h$ which corresponds an atmospheric depth $x\left(\mathrm{~g} \mathrm{~cm}^{-2}\right)$, and $F(K)\left(\mathrm{cm}^{-2} \mathrm{~s}^{-1}\right)$ is a flux of electrons with kinetic energy $K$ precipitating at the upper boundary of atmosphere. Flux of precipitating electrons at the upper boundary of atmosphere is connected with intensity of energetic particles $J(K)\left(\mathrm{cm}^{-2} \mathrm{~s}^{-1} \mathrm{sr}^{-1}\right)$ as:

$$
F(K)=\int_{0}^{2 \pi} \int_{0}^{1} J(K, \alpha, \varphi) \cos \alpha d \cos \alpha d \varphi .
$$

In our isotropic case $F(K)=1 \mathrm{~cm}^{-2} \mathrm{~s}^{-1}$ corresponding to $J(K)=1 / \pi \mathrm{cm}^{-2} \mathrm{~s}^{-1} \mathrm{sr}^{-1}$.

Note that atmospheric depth $x$ is determined such that at the upper boundary of atmosphere $(x=0)$ formally correspond to $h \longrightarrow \infty$. The functions $\rho(h), h(x)$ are presented by Tables of the Supplementary materials of this paper.

For real relativistic electron precipitation, ionization rates need to be computed for a flux of particles with different energy ranges or in other words for spectra of electron flux. In this case ionization rates must be computes as:

$$
I(h)=\int I(h, K) F(K) d K .
$$

The common scheme of computation of ionization rates by the family of CRAC (Cosmic Ray Atmospheric Cascade) models is demonstrated by Fig. 1. In this paper explanation of the scheme of computation of ionization rates is done based on the equations that discussed above. This flowchart can be applied to CRAC:CRII (Cosmic Ray Atmospheric Cascade: Cosmic Ray Induced Ionization) (Usoskin and Kovaltsov, 2006, Usoskin et al. 2010, 2011), and to vertical precipitating electrons described by the model CRAC: EPII (Cosmic Ray Atmospheric Cascade: Electron Precipitation Induced Ionization) (Artamonov et al., 2016; , Artamonov et al., 2016b).

\section{Ionization rates induced by REP with different angle distributions}

In this section we present computation results of ionization rates, $I$ (ion pairs $\mathrm{cm}^{-3}$ $\mathrm{s}^{-1}$ ), for angular distributions discussed in the previous section. All results are presented for electron flux at the upper atmosphere boundary $F=1 \mathrm{~cm}^{-2} \mathrm{~s}^{-1}$.

Figure 2 shows ionization rates vs. atmospheric altitude $(\mathrm{km})$ due to the isotropic monoenergetic electrons in the energy range $100 \mathrm{keV}-100 \mathrm{MeV}$. In top of atmosphere 


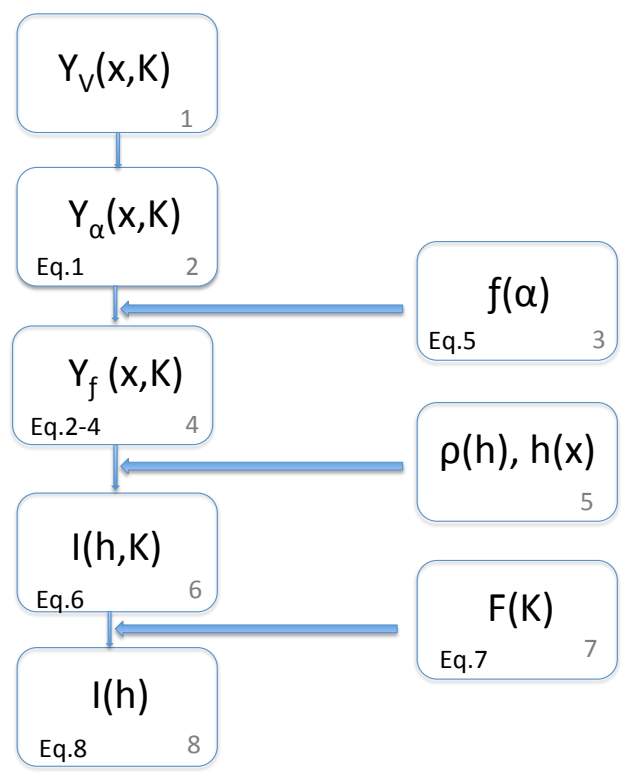

Figure 1: Flowchart of the CRAC-family (Cosmic Ray Atmospheric Cascade) models computation of ionization rates induced by: galactic cosmic rays (Usoskin and Kovaltsov 2006), solar cosmic rays (Usoskin et al. 2011), and its application to the model CRAC: EPII (Cosmic Ray Atmospheric Cascade: Electron Precipitation Induced Ionization) (Artamonov et al. 2016, Artamonov et al. 2016b).

electron flux is $1 \mathrm{~cm}^{-2} \mathrm{~s}^{-1}$. One can see that ionization rates due to isotropic case have two peaks similarly to vertical electron precipitating (Artamonov et al., 2016). The fist peak relates to the direct ionization of ambient air, the second one is ionization by bremsstrahlung. For instance, ionization profiles by REP with the energies $100 \mathrm{keV}$ (1 $\mathrm{MeV} ; 10 \mathrm{MeV} ; 50 \mathrm{MeV})$ has the first peaks in the altitude range about $80 \mathrm{~km}(60 \mathrm{~km}$; $40 \mathrm{~km} ; 30 \mathrm{~km})$ and second peaks about $37 \mathrm{~km}(30 \mathrm{~km} ; 22 \mathrm{~km} ; 17 \mathrm{~km})$. The first peaks of ionization profiles for electrons $(100 \mathrm{keV}-10 \mathrm{MeV})$ are in a good agreement with the results of analytical models (e.g. Turunen et al. (2009), see Fig.3 right panel). Note: The classical analytical models did not consider secondary bremsstrahlung radiation and scattering of primary electrons in the atmosphere (e.g. Goldberg et al. (1984)).

Figure 3 shows REP ionization rates for electrons with incidence zenith angle $\alpha: 0^{\circ}$, $15^{\circ}, 30^{\circ}, 45^{\circ}$ and for isotropic case. The behaviour of ionization rates dependences on angle of incident because with increasing the angle of the incidence of a particle, the vertical projection of the travelled distance by this particle decreases. However, the energy deposition and ionization rate integrated over altitude are the same for all incidence angles. 


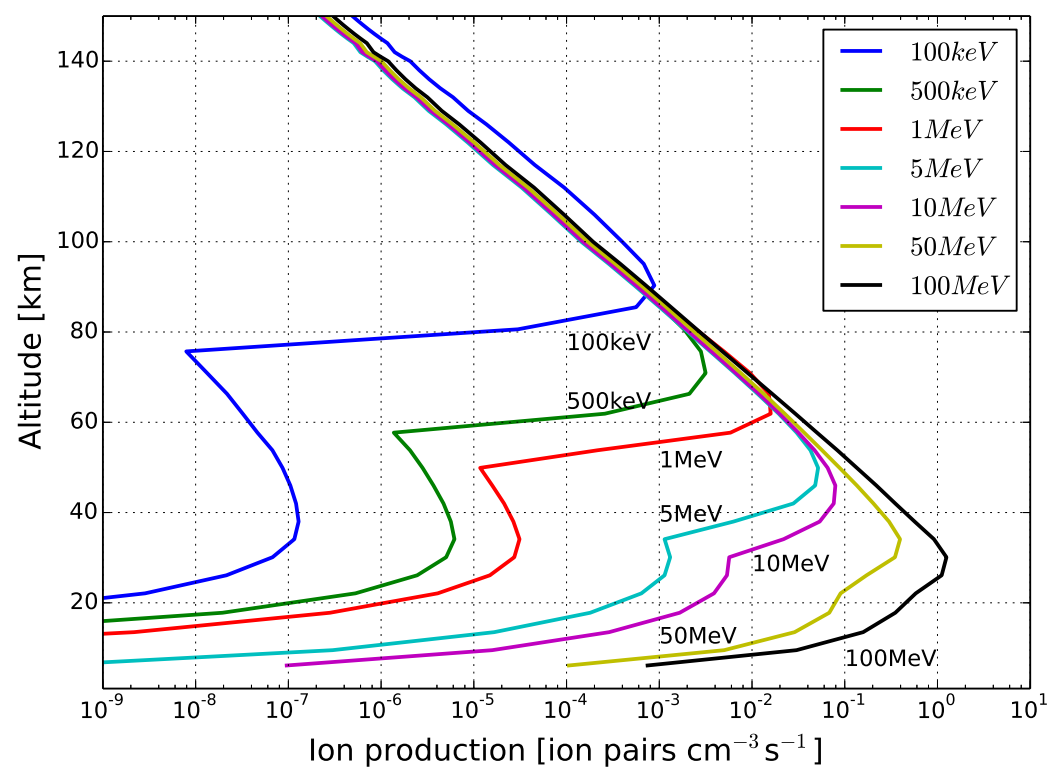

Figure 2: Ionization rates vs. altitude due to isotropic incidence of monoenergetic electrons in the energy range $100 \mathrm{keV}-100 \mathrm{MeV}$, as denoted in the legend.

For verification of our approach (eq.1) we made simulations ionization rates using GEANT 4 tool for different angles and energies of incidence electrons. Figure 4 shows a comparison of ionization rates for electrons with energies $1 \mathrm{MeV}$ and $10 \mathrm{MeV}$ and for incidence angles $\alpha: 15^{\circ}, 30^{\circ}, 45^{\circ}$. One can see a good agreement between our computations and Monte Carlo simulations. At Fig. 5 we present results of computations of the ionization rates for angular distribution function $f(\alpha)$ determined by eq. 5. The big difference in ionization rates between cases of vertical and isotropic electron precipitation is shown. A case when $n=1$ is an isotropic case and when $n \rightarrow \infty$ is a case of vertical incidence. In top of atmosphere electron flux is $1 \mathrm{~cm}^{-2} \mathrm{~s}^{-1}$. One can see that with numerical growing of numbers $n$ the curve of ionization rates tends to the curve of ionization rates in case of vertical precipitating particles.

\section{Conclusion}

In this paper we presented a scheme of computation of ionization rates induced by REP with different angle of incidence as well as isotropic distribution of relativistic electron precipitation. All computations are done for monoenergetic electrons with energy range from $100 \mathrm{keV}$ to $500 \mathrm{MeV}$. The ionization rates for precipitating electrons with $0^{\circ}$ (vertical case), $15^{\circ}, 30^{\circ}, 45^{\circ}$ angles of incidence as well as for isotropic case of REP are given as Look-up Tables 1-5, see Supplementary materials and Appendix 


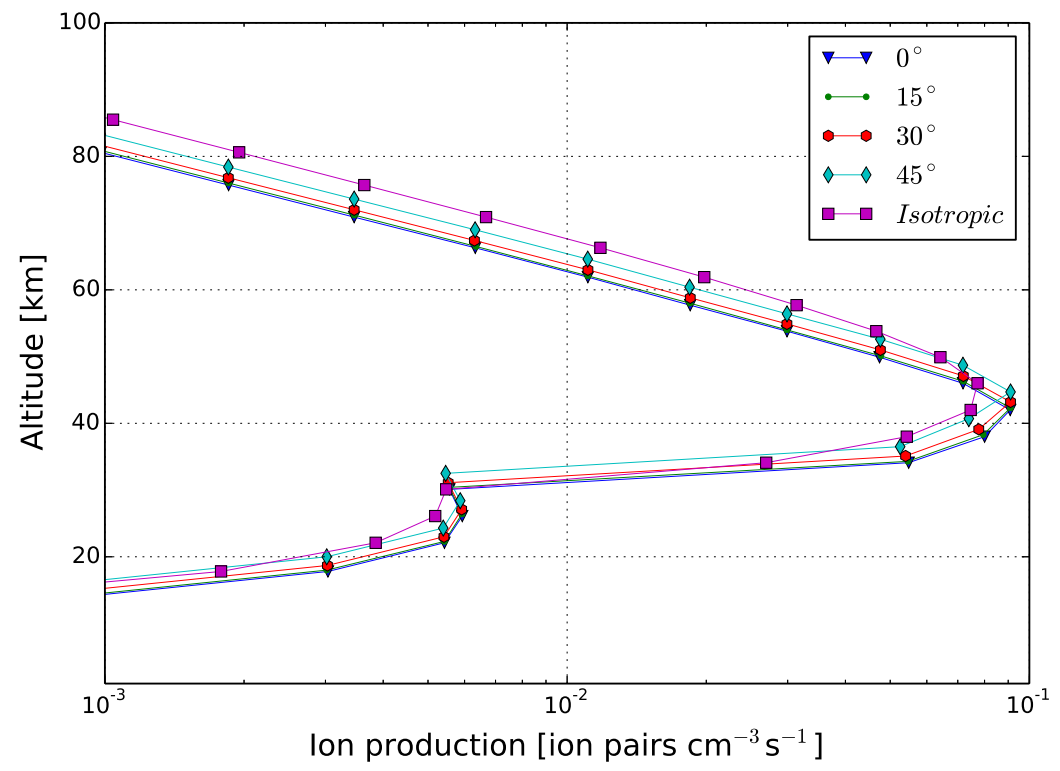

Figure 3: Ionization rates vs. atmospheric altitudes $(\mathrm{km})$ for electron with $10 \mathrm{MeV}$ energy for various angle of incidence as well for isotropic as denoted in the legend.

for explanation. Computed ionization rates induced by relativistic electron precipitation show ionization rates induced by primary relativistic electrons and ionization of bremsstrahlung.

\section{Appendix: Supplementary data. Look-up Tables (tables 1-5) of ionization rates induced by REP with an arbitrary angular distribution of precipitation electrons}

In the Supplementary materials of this paper there are five Look-up Tables with ionization rates induced by REP with vertical and arbitrary angular distribution. Tables $1-$ 5 present Look-up Tables of ionization rates $I(h)$ (ion pairs $\mathrm{cm}^{-3} \mathrm{~s}^{-1}$ ) computed per one simulated primary electron per second, with angles of incidence $0^{\circ}$ (vertical case), $15^{\circ}$, $30^{\circ}, 45^{\circ}$ and in case of isotropic electron precipitation. The ionization rates are given as a function of the atmospheric altitude $h(\mathrm{~km})$. The first column of all Look-up Tables represents atmospheric altitudes a.s.l. $h(\mathrm{~km})$. All other columns are ionization rates $I(h)$ (ion pairs $\mathrm{cm}^{-3} \mathrm{~s}^{-1}$ ) for the energies of REP from $100 \mathrm{keV}$ till $500 \mathrm{MeV}$.

\section{Acknowledgements}

The authors acknowledge ReSoLVE Centre of Excellence, Academy of Finland Rroj.272157, for their help and support of the work on this paper. IM thanks support 

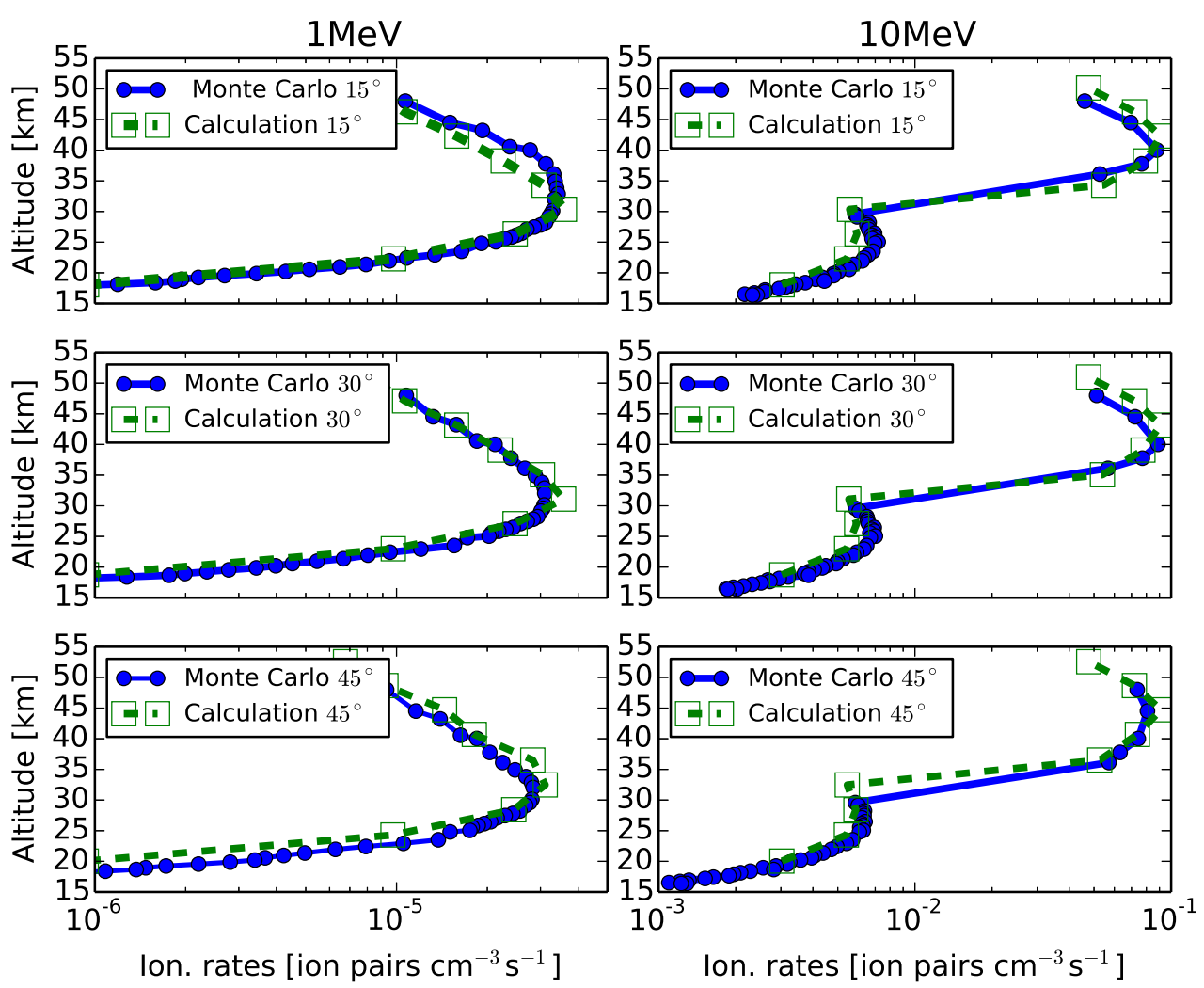

Figure 4: Comparison of ionization rates due to electrons with various angle of incidence and energy as denoted in the legend, computed with eq.1 (green squares lines) and PLANETOCOSMICS (blue dots lines). The left hand panels denote electrons with energy $1 \mathrm{MeV}$, right hand denote electrons with energy $10 \mathrm{MeV}$.

of St. Petersburg State University through the Grant 11.42.1069.2016. This work is also a part of ROSMIC WG1 activity within the SCOSTEP VarSITI program. The authors would like to acknowledge the ISSI Team members (Specification of Ionization Sources Affecting Atmospheric Processes (http://www.issibern.ch/teams/ionizationsources) for fruitful discussion and motivation to this work.

\section{References}

Artamonov, A., Mishev, A., Usoskin, I., 2016b. Atmospheric ionization induced by precipitating electrons: Comparison of crac:epii model with a parametrization model. J. Atmos. Solar-Terr. Phys. 149, 161 166.

Artamonov, A. A., Mishev, A. L., Usoskin, I. G., Feb. 2016. Model CRAC:EPII for atmospheric ionization due to precipitating electrons: Yield function and applications. J. Geophys. Res. 121, 1736-1743. 


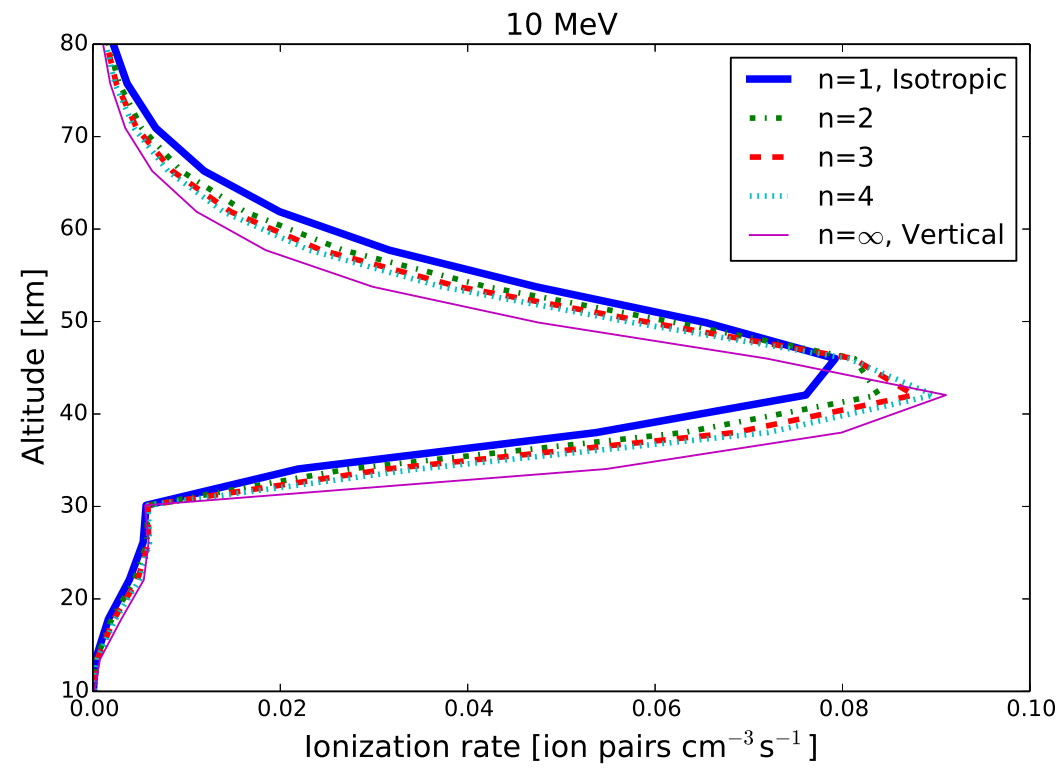

Figure 5: Dependence of computation of ionization rates on $n$ (see eq. 5. for $10 \mathrm{MeV}$ electrons.

Banks, P. M., Chappell, C. R., Nagy, A. F., Apr. 1974. A new model for the interaction of auroral electrons with the atmosphere: Spectral degradation, backscatter, optical emission, and ionization. J. Geophys. Res 79, 1459-1470.

Bazilevskaya, G. A., Usoskin, I. G., Flückiger, E. O., Harrison, R. G., Desorgher, L., Bütikofer, R., Krainev, M. B., Makhmutov, V. S., Stozhkov, Y. I., Svirzhevskaya, A. K., Svirzhevsky, N. S., Kovaltsov, G. A., 2008. Cosmic ray induced ion production in the atmosphere. Space Sci. Rev. 137, 149-173.

Cresswell-Moorcock, K., Rodger, C. J., Kero, A., Collier, A. B., Clilverd, M. A., Häggström, I., Pitkänen, T., Oct. 2013. A reexamination of latitudinal limits of substorm-produced energetic electron precipitation. J. Geophys. Res. 118, 6694-6705.

Frahm, R. A., Winningham, J. D., Sharber, J. R., Link, R., Crowley, G., Gaines, E. E., Chenette, D. L., Anderson, B. J., Potemra, T. A., Dec. 1997. The diffuse aurora: A significant source of ionization in the middle atmosphere. J. Geophys. Res. 102, 28.

Goldberg, R. A., Jackman, C. H., Barcus, J. R., Soraas, F., Jul. 1984. Nighttime auroral energy deposition in the middle atmosphere. J. Geophys. Res. 89, 5581-5596.

Kero, A., Vierinen, J., McKayBukowski, D., Enell, C., Sinor, M., Roininen, L., Ogawa, Y., 8 2014. Ionospheric electron density profiles inverted from a spectral riometer measurement. Geophys. Res. Lett. 41 (15), 5370-5375.

Makhmutov, V., Bazilevskaya, G., Stozhkov, Y., Svirzhevskaya, A., Svirzhevsky, N., 2016. Catalogue of electron precipitation events as observed in the long-duration cosmic ray balloon experiment. J. Atmos. Solar-Terr. Phys. 149, $258-276$.

Matthes, K., Funke, B., Anderson, M. E., Barnard, L., Beer, J., Charbonneau, P., Clilverd, M. A., Dudok de Wit, T., Haberreiter, M., Hendry, A., Jackman, C. H., Kretschmar, M., Kruschke, T., Kunze, M., Langematz, U., Marsh, D. R., Maycock, A., Misios, S., Rodger, C. J., Scaife, A. A., Seppälä, A., Shangguan, M., Sinnhuber, M., Tourpali, K., Usoskin, I., van de Kamp, M., Verronen, P. T., Versick, S., 2016. Solar forcing for cmip6 (v3.1). Geos. Model Develop. Discuss. 2016, 1-82. 
Mironova, I. A., Aplin, K. L., Arnold, F., Bazilevskaya, G. A., Harrison, R. G., Krivolutsky, A. A., Nicoll, K. A., Rozanov, E. V., Turunen, E., Usoskin, I. G., Nov. 2015. Energetic Particle Influence on the Earth's Atmosphere. Space Sci. Rev. 194, 1-96.

Miyoshi, Y., Oyama, S., Saito, S., Kurita, S., Fujiwara, H., Kataoka, R., Ebihara, Y., Kletzing, C., Reeves, G., Santolik, O., Clilverd, M., Rodger, C. J., Turunen, E., Tsuchiya, F., Apr. 2015. Energetic electron precipitation associated with pulsating aurora: EISCAT and Van Allen Probe observations. J. Geophys. Res. 120, 2754-2766.

Newell, P. T., Feldstein, Y. I., Galperin, Y. I., Meng, C.-I., May 1996. Morphology of nightside precipitation. J. Geophys. Res. 101, 10737-10748.

Newell, P. T., Sotirelis, T., Wing, S., Mar. 2010. Seasonal variations in diffuse, monoenergetic, and broadband aurora. J. Geophys. Res. 115, 3216.

Rees, M. H., Dec. 1969. Auroral Electrons. Space Sci. Rev. 10, 413-441.

Remenets, G. F., Astafiev, A. M., May 2015. Southern boundaries of ultraenergetic relativistic electron precipitations in several cases from 1982 to 1986 years. J. Geophys. Res. 120, 3318-3327.

Remenets, G. F., Astafiev, A. M., Sep. 2016. Solution uniquity of an inverse VLF problem: A case-study of the polar, ground-based, VLF radio signal disturbances caused by the ultra-energetic relativistic electron precipitations and of their southern boundaries. Adv. Space Res. 58, 878-889.

Shprits, Y. Y., Drozdov, A. Y., Spasojevic, M., Kellerman, A. C., Usanova, M. E., Engebretson, M. J., Agapitov, O. V., Zhelavskaya, I. S., Raita, T. J., Spence, H. E., Baker, D. N., Zhu, H., Aseev, N. A., Sep. 2016. Wave-induced loss of ultra-relativistic electrons in the Van Allen radiation belts. Nat. Commun. 7, 12883.

Turunen, E., Verronen, P. T., Seppälä, A., Rodger, C. J., Clilverd, M. A., Tamminen, J., Enell, C.-F., Ulich, T., Jul. 2009. Impact of different energies of precipitating particles on $\mathrm{NO}_{x}$ generation in the middle and upper atmosphere during geomagnetic storms. J. Atmos. Solar-Terr. Phys. 71, 1176-1189.

Usoskin, I. G., Kovaltsov, G. A., 2006. Cosmic ray induced ionization in the atmosphere: Full modeling and practical applications. J. Geophys. Res. 111, D21206.

Usoskin, I. G., Kovaltsov, G. A., Mironova, I. A., 2010. Cosmic ray induced ionization model CRAC:CRII: An extension to the upper atmosphere. J. Geophys. Res. 115, D10302.

Usoskin, I. G., Kovaltsov, G. A., Mironova, I. A., Tylka, A. J., Dietrich, W. F., 2011. Ionization effect of solar particle GLE events in low and middle atmosphere. Atmos. Chem. Phys. 11, 1979-1988. 
Supplementary Material
Click here to download Supplementary Material: AdvSpR.xls

Supplementary Material
Click here to download Supplementary Material: AdvSpR.xls 